\title{
Isolation and genomic characterization of Sfl, a serotype-converting bacteriophage of Shigella flexneri
}

Qiangzheng Sun ${ }^{1 \dagger}$, Ruiting Lan ${ }^{2 \dagger}$, Yiting Wang ${ }^{1 \dagger}$, Jianping Wang ${ }^{1 \dagger}$, Yan Wang ${ }^{1}$, Peijing Li ${ }^{1}$, Pengcheng Du ${ }^{1}$ and Jianguo $\mathrm{Xu}^{1 *}$

\begin{abstract}
Background: All Shigella flexneri serotypes except serotype 6 share a common O-antigen tetrasaccharide backbone and nearly all variations between serotypes are due to glucosyl and/or O-acetyl modifications of the common $\mathrm{O}$ unit mediated by glycosyltransferases encoded by serotype-converting bacteriophages. Several $S$. flexneri serotype-converting phages including SfV, SfX, Sf6 and Sfll have been isolated and characterized. However, S. flexneri serotype-converting phage Sfl which encodes a type I modification of serotype 1 (1a, 1b, 1c and 1d) had not yet been characterized.

Results: The Sfl phage was induced and purified from a S. flexneri serotype 1a clinical strain 019. Electron microscopy showed that the Sfl phage has a hexagonal head and a long contractile tail, characteristic of the members of Myoviridae family. Sfl can convert serotype $Y$ to serotype $1 \mathrm{a}$ and serotype $X$ to serotype $1 \mathrm{~d}$, but cannot convert 10 other $\mathrm{S}$. flexneri serotypes (1a, 1b, 2a, 2b, 3a, 3b, 4a, 4b, 5a, Xv) tested, suggesting that Sfl has a narrow host range. Similar to other $S$. flexneri serotype-converting phages, $\mathrm{Sfl}$ integrates into the tRNA-thrW gene adjacent to proA of the host chromosome when lysogenized. The complete sequence of the Sfl genome was 38,389 bp, encoding 66 open reading frames and two tRNA genes. Phage Sfl shares significant homology with S. flexneri phage SfV, Escherichia coli prophage e14 and lambda, and is classified into the lambdoid phage family. Sfl was found to use a cos mechanism for DNA packaging similar to that of phage SFV.
\end{abstract}

Conclusions: Sfl contains features of lambdoid phages and is closely related to S. flexneri phage SfV, E. coli prophage e14 and lambda. The characterization of $\mathrm{Sfl}$ enhances our understanding of serotype conversion of S. flexneri.

\section{Background}

Shigella is the major cause of endemic bacillary dysentery (shigellosis) in developing countries. It is estimated that there are about 164.7 million cases of shigellosis annually worldwide, of which 163.2 million were in developing countries, resulting in 1,1 million deaths, most of which were children under 5 years of age [1]. Among the four Shigella species, S. dysenteriae, S. flexneri, S. boydii, and S. sonnei, S. flexneri is the predominant species.

Based on the combination of antigenic determi nants present in the $\mathrm{O}$-antigen of the cell envelope

\footnotetext{
* Correspondence: xujianguo@icdc.cn

${ }^{+}$Equal contributors

'State Key Laboratory for Infectious Disease Prevention and Control, National Institute for Communicable Disease Control and Prevention, China CDC, P.O. Box 5, Beijing, Changping, China

Full list of author information is available at the end of the article
}

lipopolysaccharide (LPS), S. flexneri is further divided into various serotypes. To date, at least 16 serotypes have been recognized [2-4]. Except for serotype 6, all share a basic repeating tetrasaccharide unit, comprised of one GlcNAc and three rhamnoses [4]. Modifications to the side chain of the tetrasaccharide by the addition of glucosyl and/or $O$-acetyl groups give rise to various antigenic determinants [3]. The genes responsible for the $\mathrm{O}$-antigen modification are always either the gene cluster gtrABC for glucosyl groups or the single oac gene for the $O$-acetyl group; all encoded by serotypeconverting bacteriophages [3,5-10]. In all glucosylation modification phages, the gtrABC gene cluster is always located immediately upstream of the attP site, followed by the int and xis genes [6].

$\mathrm{Up}$ to now, four $S$. flexneri serotype-converting bacteriophages, SfV, SfX, Sf6 and SfII, have been induced

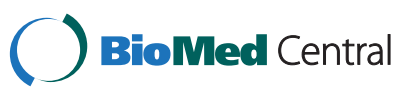


and purified by different groups [8,11-13]. Morphologically, SfV and SfII, which have an isometric head and a long tail, belong to Group A in the family of Myoviridae $[8,11]$; while SfX and Sf6, which possess a short tail linked to an isometric head, belong to the family of Podovirida [12,13]. The complete genome sequences of phage SfV and Sf6 have been obtained by directly sequencing the phage DNA purified from phage particles, and their genetic features have been well characterized $[9,10]$. Recently, the prophage genome of SfX was determined from the sequenced S. flexneri serotype Xv strain 2002017; which is presumably the whole genome of phage SfX, because a SfX phage particle can be induced and isolated from 2002017 [2]. The SfX genome is 37,355 bp length, encoding 59 ORFs (unpublished data). The genome of SfII has not yet been sequenced from free phage particles, but prophage genomes can be derived from sequenced $S$. flexneri serotype 2a strains Sf301 and 2457T [14,15], which show considerable variation with one or both being prophage remnants.

S. flexneri serotype 1 is defined by reaction with type I antisera. A total of 4 subtypes, 1a, 1b, 1c and 1d have been recognized [16-18]. In serotype 1, a glucosyl group is attached to the GlcNac residue of the repeating unit by an alpha-1, 4 linkage, which results in the presence of serotype 1-specific I antigen. The type I modification is mediated by an O-antigen glucosylation locus ( $g$ trI, gtrA, gtrB) encoded on the SfI prophage genome [5]. The glucosylation genes and flanking partial SfI sequences were previously obtained from a serotype 1a strain Y53 [17]. However, the free phage particle of SfI had not been isolated, and its full genomic characteristics have not yet been elucidated [5].

In this study, we induced and purified the free SfI phage particles from $S$. flexneri serotype 1a clinical strain 019 and characterized its morphology, host range and genomic features.

\section{Results and discussion Isolation of phage Sfl from S. flexneri serotype 1a strain 019}

Using the conditions described in Methods, we induced the SfI phage from serotype 1a strain 019. Plaques were observed on the semi-solid LB agar when the host strain 036 was infected with induced products from strain 019 . Lysogens isolated from plaques were serologically identified as serotype 1a, characterized by agglutination with both typing sera I and grouping sera 3;4. PCR amplification indicated that the SfI specific gene gtrI is present on both phage particles and the lysogens. These results suggest that phage SfI has been successfully induced and isolated from strain 019. This is the first report of isolation of free SfI particles from S. flexneri.
The morphology of $\mathrm{Sfl}$ is characteristic of the Myoviridae family

The purified SfI phage particles were morphologically analyzed using electron microscopy. The phage has a hexagonal head of ca. $55 \mathrm{~nm}$ in diameter, a knob-like neck, a contractile tail of ca. $110 \mathrm{~nm}$, and a tail sheath of ca. $55 \mathrm{~nm}$ (Figure 1). There are indications of a baseplate-like structure and long tail fibers, but no other distinctive features could be seen (Figure 1). These characteristics suggest that phage SfI is a member of the Myoviridae family in the order Caudovirale [19].

In comparison to other morphologically characterized serotype-converting phages Sf6, SfV, SfII and SfX, SfI has a very similar appearance to SfII and SfV $[8,11]$, but distinctive from SfX and Sf6 [12,20]. The microscopic difference reflected the genetic divergence among them in that the SfI packaging and structure genes were identical to those of phage $\mathrm{SfV}$, but divergent from those of SfX and Sf6 (see below, Figure 2).

\section{Phage Sfl has a very narrow host range}

Host specificity of serotype-converting bacteriophages has long been recognized, which results in the specific lytic spectrum and serotype conversion of S. flexneri in nature [20]. The recognition between the O-antigen of host bacterium and the tail component of a phage is the key mechanism of host specificity [20]. To determine the host range of SfI, 132 S. flexneri strains of 12 serotypes (1a, 1b, $2 \mathrm{a}, 2 \mathrm{~b}, 3 \mathrm{a}, 3 \mathrm{~b}, 4 \mathrm{a}, 4 \mathrm{~b}, 5 \mathrm{a}, \mathrm{Y}, \mathrm{X}$ and $\mathrm{Xv}$ ) were tested following the methods described in the Methods. Apart from 10 serotype $\mathrm{Y}$ strains, which were all converted to serotype $1 \mathrm{a}$ as expected, the 24 serotype $\mathrm{X}$ strains tested

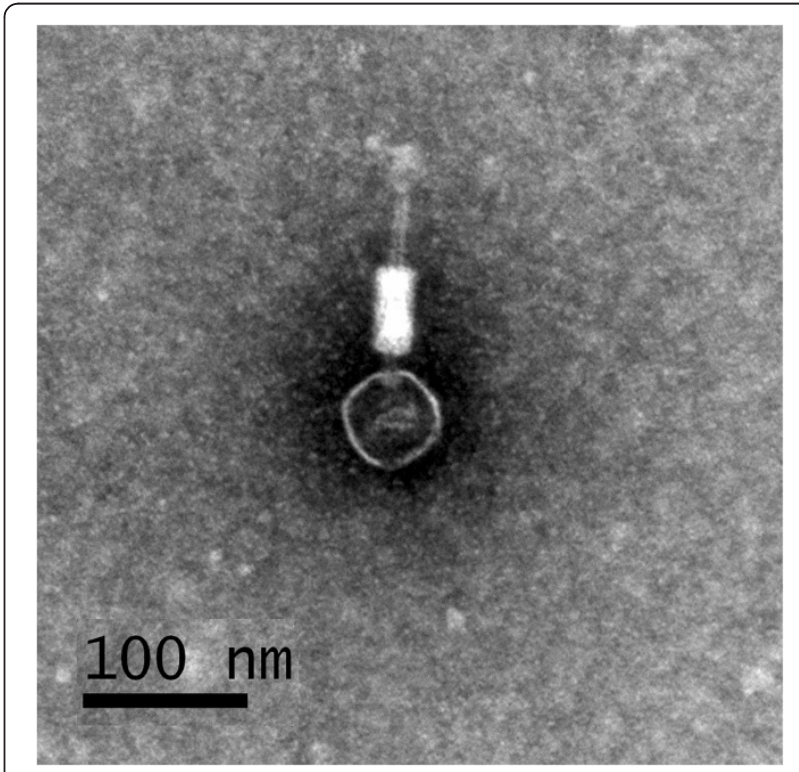

Figure 1 Electron micrograph of S. flexneri bacteriophage Sfl stained with phosphotungstic acid. 


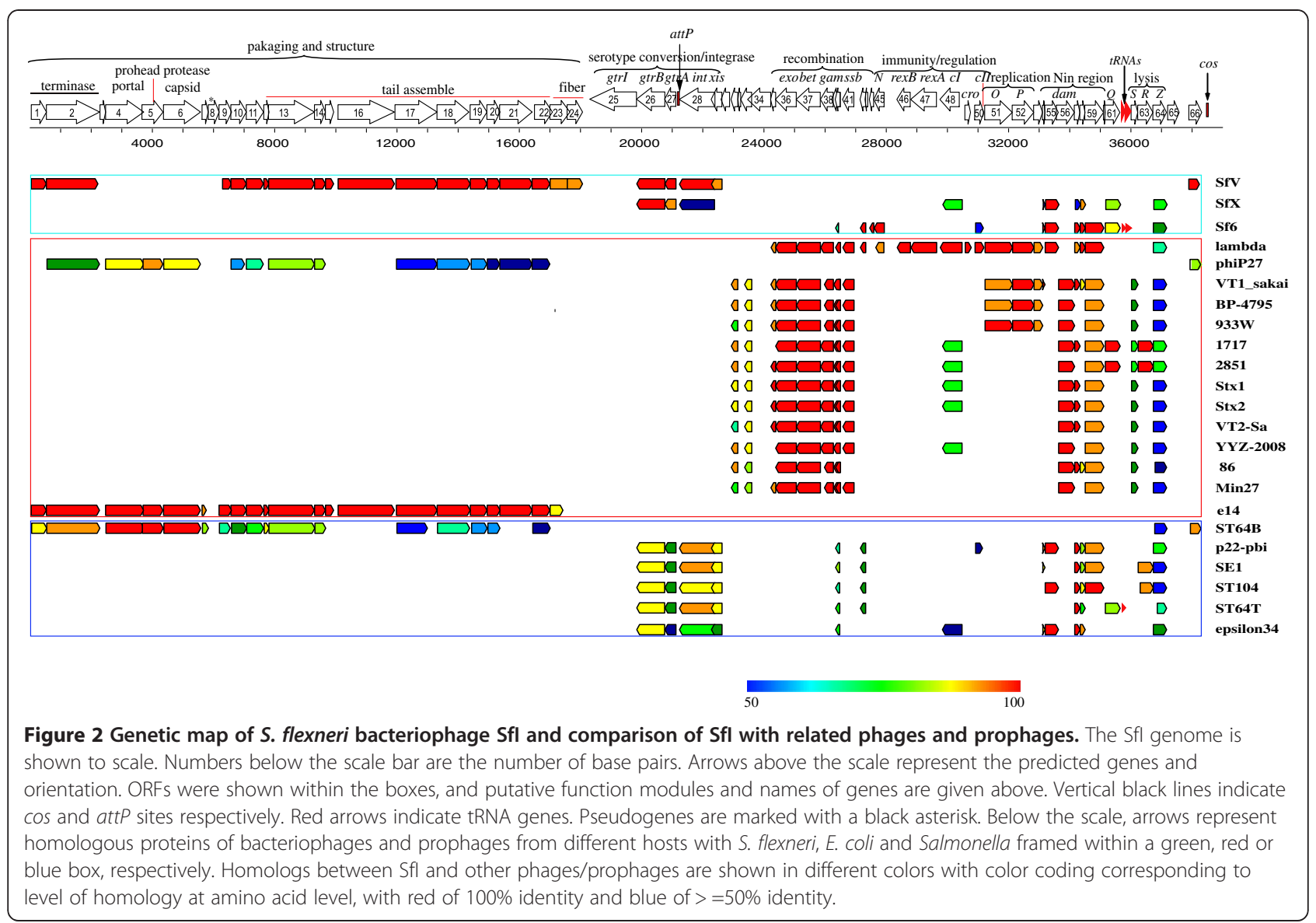

were also lysogenized, and converted to a newly named serotype $1 \mathrm{~d}$ [16]. The serotype $1 \mathrm{~d}$ strains were serologically characterized as reacting with both serotype 1 specific I typing sera and serotype $\mathrm{X}$ specific $7 ; 8$ grouping sera [16]. Interestingly, such a serotype has already appeared in natural infections in Anhui and Henan provinces, China [21]. Except for serotypes $\mathrm{Y}$ and $\mathrm{X}$, the other serotypes could not be lysogenized by phage SfI. A possible explanation for the host range restriction of phage SfI is phage immunity due to modification of the $\mathrm{O}$-antigen as phage receptors [22].

Sfl uses a site-specific mechanism for DNA packaging and has the same attP core sequence as Sfll, SfIV, SfV and SfX Restriction enzyme analysis revealed that phage SfI has a linear but not circular genome (data not shown). Genomic comparison found that the SfI prophage genome has similar packaging genes to that of phage $\mathrm{SfV}$; and the fragments adjacent to them were also highly similar to the cohesive end site ( $\cos$ ) of phage SfV [9], with only one base difference at the $5^{\prime}$ end ( $\mathrm{T}$ versus $\left.\mathrm{A}\right)$. These data suggest that SfI may use the same site-specific mechanism as SfV for packaging. Direct sequencing of the putative termini of the SfI genome extracted from free phage particles and comparison of the corresponding regions with the SfI prophage genome in strain 019 revealed a 10 nucleotide $\left(5^{\prime}\right.$ TGCCCGCCCC $-3^{\prime}$ ) gap in the SfI phage genome. Therefore, we conclude that SfI uses a cos mechanism for DNA packaging as postulated for phage SfV [9], and does not use a head full mechanism ( $p a c)$ as for phage Sf6 and SfX $[10,12]$.

Integration of lambdoid phages into the bacterial chromosome generally occurs by site-specific recombination between the phage attP and the bacterial attB sites [23]. In all serotype-converting phages except for Sf6, the attP site is always found located immediately downstream of the O-antigen modification genes, and preceded by the int and xis genes [6]. To determine the attP site of phage SfI, the region between genes gtrA and intI of SfI was PCR amplified and sequenced and a 261 bp sequence was obtained, in which, 46 bases, ATTCGTAATGCGAAGGTCGTAGGTTCGACTCCTAT TATCGGCACCA, were found to be identical to the attR/attL core sequence of prophage SfI in strain Y53 [5] (Figure 3). In the lysogen of 036_1a, the 261 nucleotide sequence was divided into two parts, located at opposite ends of the SfI prophage genome (Figure 3). Evidently, site-specific recombination occurred at this attP 
site. The attP core sequence of SfI is identical to that of $S$. flexneri serotype-converting phage SfII, SfV and SfX, as well as that of serotype-converting phages p22 of Salmonella typhimurium and DLP12 of E. coli $[5,8,24]$.

\section{Characterization of Sfl genome sequence}

The complete genome sequence of SfI was obtained by combining the SfI prophage genome of host strain 019 with the attP site obtained by PCR sequencing as above. Firstly, the whole genome sequence of host strain 019 was sequenced using Illumina Solexa sequencing. A total of $4,382,674$ reads were generated to reach about 110 -fold coverage and assembled de novo into 376 contigs and scaffolds. The SfI prophage genome located between genes int and gtrIA was extracted from one of the contigs which was further assembled with the attP site sequence obtained above to construct a circular phage SfI genome. To revert to the linear organization as usual practice, we artificially linearised the sequence starting from the terminase small subunit gene and ending with the cos site (Figure 2).

The genome size of SfI is 38,389 bp similar to that of sequenced $S$. flexneri serotype-converting phages Sf6 $(39,043$ bp) [9], SfV (37,074 bp) [10] and SfX $(37,355)$ (unpublished data). The overall G $+\mathrm{C}$ content is $50.12 \%$, which is very similar to that of its host (50.9\%) [25]. Sixty-six putative ORFs (including one pseudogene) were predicted and their functions are listed in the Additional file 1: Table S1.

The genetic architecture of the SfI genome is similar to that of sequenced $S$. flexneri serotype-converting phages SfV, Sf6 and SfX: the left-most region encodes genes for phage packaging and structure, followed by the middle region with genes involved in serotype conversion, integration/excision, recombination, immunity and regulation, replication and the Nin region, and then the putative lysis cassette at the right-most region ending with the cos site of the phage genome (Figure 2). The genomic structure of SfI is also similar to that of phage SfV and lambda. Thus it belongs to the family of lambdoid phages.

tRNAscan was used to find tRNA genes. Two tRNA genes in tandem, with anticodons GUU for asparagine (Asn) and UGU for threonine (Thr), were found to be located downstream of gene Q (35,738 - 35,809 for Asn, and 35,818 - 35,890 for Thr). One or both of these tRNA genes were also to be found located at this position in phage Sf6, ST64T, PS3 and p21 [10,26,27]. A recent study

$<$ gtrlA $<<$

TACAAATAACTTTAACATTTATTTAATCAATGAGCTCTGAAAGGCATGAAGTCTATCATCCAAGTCTCA

ATTGATCGATACTTGCTATGTCTGATGAGACAAAACTGAGACACATAAGGCCTCACAATGGCTTGCAA

A GGCTTTACATGTTTTGATGTGGTGGGACGTGTGAGCGCAGTGTTGATGGGATAATCCTTTGAATTACA AGCGGATTCTTATAATTCGTAATGCGAAGGTCGTAGGTTCGACTCCTATTATCGGCACCATTAAAATCA ATAAGTTACACATCATTAGTACCTTC

$<$ intl $<$

$>\operatorname{proA}>>$

ACCATTCGTGCGTAA ATAAAACCGGGTGATGCAAAAGCAGCCATTTGATTCACAAGGCCATTGACGCA

B TCGCCCGGTTAGTTTTAACCTTGTCCACCGTGATTCACGTTCGTGAACATGTCCTTTCAGGGCCGATAT AGCTCAGTTGGTAGAGCAGCGCATTCGTAATGCGAAGGTCGTAGGTTCGACTCCTATTATCGGCACCA TTTAAATCAATAAGTTACACATCATTAGTACCTTC

$<$ putative phage int $<$

$>\operatorname{proA}>>$

ACCATTCGTGCGTAAATAAAACCGGGTGATGCAAAAGCAGCCATTTGATTCACAAGGCCATTGACGCA

C TCGCCCGGTTAGTTTTAACCTTGTCCACCGTGATTCACGTTCGTGAACATGTCCTTTCAGGGCCGATAT AGCTCAGTTGGTAGAGCAGCGCATTCGTAATGCG $<$ intl $<<$

$<$ gtrlA $<<$

TAACTTTAACATTTATTTAATCAATGAGCTCTGAAAGGCATGAAGTCTATCATCCAAGTCTCAATTGATC GATACTTGCTATGTCTGATGAGACAAAACTGAGACACATAAGGCCTCACAATGGCTTGCAAGGCTTTA

D CATGTTTTGATGTGGTGGGACGTGTGAGCGCAGTGTTGATGGGATAATCCTTTGAATTACAAGCGGAT TCTTATAATTCGTAATGCGAAGGTCGTAGGTTCGACTCCTATTATCGGCACCATTTAAATCAATAAGTTA CACATCATTAGT

$<<$ putative phage int $<<$

Figure 3 DNA sequences of chromosomal integration site of $\mathbf{S}$. flexneri phage Sfl. Sequences obtained by PCR and sequencing of junction regions using a series of primers across the integration site. (A) attP in phage Sfl. (B) attB in strain 036. (C) attL in strain 036_1a. (D) attR in 036_1a. Sequences in box are DNA regions between conserved genes; Underlined sequences are tRNA-thrW; Sequences in blue are att core sequence; Conserved genes are shaded and their transcription orientation is marked by an arrow. 
suggested that phage-encoded tRNA could serve to supplement the host tRNA reservoir, allowing the rare codons in the phage to be more efficiently decoded [28]. Codon analysis indeed found a convincing bias of ACA (anticodon UGU) in the SfI genome when compared to its $S$. flexneri host (with $17.3 \%$ in phage SfI, and $7.1 \%$ in strain Sf301), but no obvious bias was observed on CAA (anticodon GUU), and the significance of the $t R N A-A s n$ in SfI is not clear.

Genomic comparison reveals that $\mathrm{Sfl}$ is genetically related to Shigella phage SfV, E. coli prophage e14 and lambda

The ORFs encoded in the SfI genome were searched against the GenBank database at both DNA and amino acid levels. SfI encoded proteins exhibited homology to various phages and prophages originating from various hosts, including Shigella (SfV, Sf6 and SfX), E. coli (lambda, phip27, VT1-sakai, BP-4795, 933 W, 1717, 2851, Stx1, Stx2, VT2-Sa, YYZ-2008, 86, M27 and e14) and Salmonella (ST64B, p22-pbi, SE1, ST104, ST64T and epsilon34). Figure 2 displays the homologies of phage SfI to other phages. The SfI genes involved in phage packaging and morphogenesis are homologous and organized in a similar manner to those of phage SfV, phi-p27, ST64B and prophage e14. As reported earlier [6], the O- antigen modification and integration and excision modules (gtrA, gtrB, int and xis) are homologous to that of serotype-converting bacteriophages from S. flexneri ( $\mathrm{SfV}$ and SfX) and Salmonella (p22-pbi, SE1, ST104, ST64T and epsilon34). However, the early and regulatory regions located in the right half of the genome were homologous to that of lambda and Shiga toxin-1 and Shiga toxin-2 phages (phip27, VT1-sakai, BP4795, 933 W, 1717, 2851, Stx1, Stx2, VT2-Sa, YYZ-2008, 86 and M27).

Therefore SfI is a mosaic phage with its left half most homologous to phage $\mathrm{SfV}$ (91.6\% - 100\% identity at protein level, and $89-98 \%$ at DNA level [ORF by ORF comparison]) and E. coli prophage e14 (94.0\% - 100\% identity at protein level, and $97 \%$ at DNA level) and right half most homologous to Lambda (67\% - 100\% identity at protein level, and 80 - $98 \%$ at DNA level). Homology to SfV encompasses at least 23 ORFs encoding functions for morphology (orf1, orf2 and orf9 - orf24), O-antigen modification (orf26, orf27), integration/excision (orf28 to orf29) (Figure 2, Table 1). The homologous ORFs are located in four contiguous regions, amounting to $17,487 \mathrm{bp}$ nucleotides and accounting for $45.6 \%$ of the entire phage genome (Table 1). SfI also shared genetic relatedness with the E. coli prophage e14. The homologous regions mainly encode proteins responsible for phage assembly and morphogenesis and are located in the left half of the SfI genome (Figure 2 and Table 1). The homologous regions account for $46 \%$ of the SfI genome. Based on the homology of the first 22 ORFs (Additional file 2: Figure S1), it seems that $\mathrm{SfI}$ is closer to e14 than to $\mathrm{SfV}$ since $5 \mathrm{ORFs}$ (SfI orf3 to orf7) are highly homologous between SfI and e14, but share little homology between SfI and SfV. For the remaining 17 ORFs except orf8, the pairwise percentage identities are very similar between SfI, SfV and e14. On the other hand, the homology between SfI and SfV extends further to orf 28 with high homology of orf 23 , orf 24 and orf 26 to orf 28 . Similarly, six contiguous DNA segments, which account for $28.4 \%$ of the SfI genome, were found to be homologous to the corresponding regions of lambda. These homologous regions are mainly located in the early and regulatory regions, and encode functional modules for phage recombination (orf35 to orf43), immunity and regulation (orf45 to orf50), replication (orf51, orf52), Nin region (orf53 to orf55, orf57 to orf60), and part of the lysis module (orf64) (Figure 2 and Table 1). Thus a total of $72.9 \%$ of the SfI genome is homologous to either SfV, e14 or lambda.

\section{Conclusions}

The serotype-converting bacteriophage SfI was isolated from a $S$. flexneri serotype 1a strain. It had a narrow lytic pattern and converted only serotype $\mathrm{Y}$ to serotype 1a and serotype X to serotype 1d. Morphologically SfI is a member of the Myoviridae family in the order of Caudovirale. Genomic analysis revealed that SfI contains features of lambdoid phages and is closely related to S. flexneri phage SfV, E. coli prophage e14 and lambda. The characterization of serotype-converting phage SfI enhances our understanding of serotype conversion of S. flexneri.

\section{Methods}

\section{Bacterial strains, media and culture}

S. flexneri serotype 1a strain 019 [16] was used as the source for induction of phage SfI. S. flexneri strain 036 (serotype Y) was used as the host for phage infection and large volume propagation of SfI [16]. One hundred and thirty two $S$. flexneri strains of 12 serotypes (17 serotype 1a, 5 serotype $1 \mathrm{~b}, 10$ serotype $2 \mathrm{a}, 10$ serotype $2 \mathrm{~b}, 10$ serotype 3a, 2 serotype $3 \mathrm{~b}, 5$ serotype $4 \mathrm{a}, 5$ serotype $4 \mathrm{~b}$, 4 serotype $5 \mathrm{a}, 10$ serotype $\mathrm{Y}, 24$ serotype $\mathrm{X}$ and 30 serotype $\mathrm{Xv}$ ) were used for phage host range detection. All S. flexneri strains used in this study were isolated from diarrheal patients in China, or purchased from National Collection of Type Cultures (NCTC), UK. S. flexneri strains were serologically identified using Shigella antisera Kits (Denka Seiken, Japan) and monoclonal antibody reagents (Reagensia AB, Sweden). S. flexneri strains were routinely cultured on LB agar or in LB broth with shaking at $37^{\circ} \mathrm{C}$.

\section{Induction of phage $\mathrm{Sfl}$}

Induction of phage SfI was performed as methods described by Mavris et al. [8]. Briefly, a freshly grown colony of strain 019 was incubated in $10 \mathrm{ml} \mathrm{LB}$ broth 
Table 1 Homology of Sfl to S. flexneri phage SfV and E. coli prophage e14 and lambda

\begin{tabular}{lllll}
\hline Phage or prophage & Nucleotide position & $\begin{array}{l}\text { Homologous nucleotide position } \\
\text { in Sfl (total length [bp]) }\end{array}$ & $\begin{array}{l}\text { \% identity at } \\
\text { nucleotide level }\end{array}$ & $\begin{array}{c}\text { Sfl ORFs }^{\boldsymbol{a}} \\
\text { \% of Sfl } \\
\text { genome }\end{array}$ \\
\hline SfV & $9-2,211$ & $2-2,194(2,193)$ & 98 & orf1, (orf2) \\
& $5,793-17,782$ & $6,053-18,042(11,990)$ & 97 & orf9 - orf24 \\
(orf26), orf2 - orf29, attP & (orf66) \\
& $19,146-22,042$ & $19,787-22,681(2,895)$ & 98 & (orf31), orf32, (orf33) \\
& $36,666-37,074$ & $37,964-38,372(409)$ & 89 & (orf35), orf36- orf43 \\
Lambda & $30,418-30,910$ & $23,002-23,493(491)$ & 95 & (orf45) \\
& $31,206-34,381$ & $24,281-27,456(3,176)$ & 98 & orf46- orf55 \\
& $35,104-35,386$ & $27,708-27,990(283)$ & 98 & orf57- orf59, (orf60) \\
& $35,496-41,084$ & $28,052-33,640(5,590)$ & 98 & (orf64) \\
e14 & $42,097-43,068$ & $2-2,194(2,193)$ & 97 & orf1 - orf22, (orf66) \\
\hline
\end{tabular}

${ }^{a}$ Parentheses indicate that the region of homology starts or ends within an ORF.

${ }^{b}$ E. coli $\mathrm{S} 88$ strain genome (accession no. CU928161).

overnight with vigorous shaking. After being induced for $30 \mathrm{~min}$ at $56^{\circ} \mathrm{C}$ with aeration, the cultures were centrifuged, and the supernatants were filtered through a $0.22 \mathrm{~mm}$ membrane filter (Promega) to remove bacterial cells. The filtrates were either used directly for phage infection assay or stored at $4{ }^{\circ} \mathrm{C}$ with addition of $10 \%(\mathrm{v} / \mathrm{v})$ chloroform.

\section{Phage infection and lysogenization}

S. flexneri strain 036 cells were prepared using the methods for phage lambda [29]. Phage infection and lysogenization were performed using the methods described previously [16]. The serotypes of isolated colonies were identified by slide agglutination assay. Large volume phage purification was performed on S. flexneri strain 036, according to the methods for phage SfII [8].

\section{Electron microscopy}

The purified phages were absorbed on carbon-coated copper grids (300 mesh) and negatively stained with $2 \%$ $(\mathrm{w} / \mathrm{v})$ sodium phosphotungstate $(\mathrm{pH}$ 7.0). Samples were visualized with a Hitachi 600 electron microscope at $80 \mathrm{kV}$.

\section{Host range detection}

To determine the host range of phage SfI, one hundred and thirty two S. flexneri strains of 12 serotypes were infected with SfI. The preparation of component cells, phage infection and lysogen isolation were performed as methods for strain 036 above. The SfI host range was determined by observing the presence of plaques and serologically identification of the lysogens.

\section{Identification of the chromosomal integration site and} cohesive ends (cos sites) of phage Sfl

Oligonucleotide primers gtrI-F (5'- ATTGAACGCCTCC TTGCTATGC $\left.-3^{\prime}\right)$, intI-R ( $5^{\prime}$ - AGTGTTACAGGAA
ATGGGAGGC $\left.-3^{\prime}\right)$, proA-F ( $5^{\prime}$ - ACAAAGCGAAATCA TCCTCAA - $3^{\prime}$ ), and yaiC-R (5'- GCAGGAAACCACC ATCAACACC $-3^{\prime}$ ), which are complementary to the genes gtrI and intI in phage SfI, and proA and yaiC in $S$. flexneri chromosome, respectively, were used to identify the $a t t P$ and $a t t B$ sites of phage SfI and strain 036, as well as the $a t t R$ and $a t t L$ regions of the SfI lysogen. PCR was conducted using the Sensoquest labcycler PCR System (SENSO, German) under standard protocol. The PCR products were either cloned into TA vector PMD20$\mathrm{T}(\mathrm{TaKaRa})$ for sequencing or sequenced directly.

To determine the cohesive ends of the SfI phage, two primers, $\cos$-F: $5^{\prime}$ - ATGCCACCACGAACCCCAAAAG $-3^{\prime}$ (nt 37,964 - 37,985, complementary to SfI genome sequence), cos-R: $5^{\prime}$ - GGCTTGGGGCGACGCCCGGA - $3^{\prime}$ (nt 72-91, complementary to SfI genome), were designed to sequence the putative termini of the SfI genome directly using phage DNA as the template. The phage genome ends obtained were further compared to the corresponding regions of the SfI prophage genome in strain 019. The missing region in the former sequence is the putative cos site of phage SfI.

\section{Genome sequencing and analysis}

To obtain the entire phage genome sequence of SfI, the whole genome of source strain 019 was sequenced by Illumina Solexa sequencing. A paired-end (PE) library with an average insertion length of between 500 bp and 2,000 bp was constructed. Reads were generated with Illumina Solexa GA IIx (Illumina, San Diego, CA) and assembled into scaffolds using SOAP denovo (Release1.04). The sequence between genes intI and gtrA was extracted for further analysis. By assembling with the sequence amplified from SfI DNA using primer pair gtrI-F and int-R mentioned above, the entire sequence of SfI genome in its circular state was obtained. Open reading frames (ORFs) of 
SfI were determined using the ORF Finder program, which is accessible through the National Center for Biotechnology Information (NCBI). Searches for homologous DNA and protein sequences were conducted with the BLAST software against the non-redundant GenBank database (http://www.ncbi.nlm.nih.gov/blast/blast/). tRNA genes were determined with tRNAscan-SE Search server (http://lowelab.ucsc.edu/tRNAscan-SE).

\section{Nucleotide accession number}

The genomic sequence of phage SfI has been deposited in GenBank as accession number JX509734.

\section{Additional files}

Additional file 1: Table S1. Analysis of predicted ORFs and proteins of Sfl. Additional file 2: Figure S1. Gene by gene comparison of homologous regions of Sfl with S. flexneri phage SfV and E. coli prophage e14. The arrows indicate the predicted proteins and orientation of the ORFs. The regions marked with a lightly red rectangle represent $>50 \%$ sequence identity at amino acid level.

\section{Competing interests}

The authors declare that they have no competing interest.

\section{Authors' contributions}

JX, QS and RL designed the study, and co-drafted the manuscript. YW participated in the induction of the phage. JW carried out the PCR amplification and DNA sequencing. PL participated in the phage induction and infection. YW and PD participated in the sequence alignment and genome annotation. All authors read and approved the final manuscript.

\section{Acknowledgements}

This work was supported by grants from the National Natural Science Foundation of China (No. 81271788), the National Basic Research Priorities Program (2011CB504901), the Project of State Key Laboratory for Infectious Disease Prevention and Control (2011SKLID203, 2008SKLID106), the National Key Program for Infectious Diseases of China (2013ZX10004221, 2013ZX10004216-001-002) and the Special Project of Beijing Educational Committee (YB20098450101)

\section{Author details}

'State Key Laboratory for Infectious Disease Prevention and Control, National Institute for Communicable Disease Control and Prevention, China CDC, P.O Box 5, Beijing, Changping, China. ${ }^{2}$ School of Biotechnology and Biomolecular Sciences, University of New South Wales, Sydney, NSW 2052, Australia.

Received: 24 August 2012 Accepted: 31 January 2013

Published: 17 February 2013

\section{References}

1. Kotloff KL, Winickoff JP, Ivanoff B, Clemens JD, Swerdlow DL, Sansonetti PJ, Adak GK, Levine MM: Global burden of Shigella infections: implications for vaccine development and implementation of control strategies. Bull World Health Organ 1999, 77(8):651-666.

2. Ye C, Lan R, Xia S, Zhang J, Sun Q, Zhang S, Jing H, Wang L, Li Z, Zhou Z, et al: Emergence of a new multidrug-resistant serotype $X$ variant in an epidemic clone of Shigella flexneri. J Clin Microbiol 2010, 48(2):419-426.

3. Stagg RM, Tang SS, Carlin NI, Talukder KA, Cam PD, Verma NK: A novel glucosyltransferase involved in O-antigen modification of Shigella flexneri serotype 1c. J Bacteriol 2009, 191(21):6612-6617.

4. Simmons DA, Romanowska E: Structure and biology of Shigella flexneri O antigens. J Med Microbiol 1987, 23(4):289-302.
5. Adhikari P, Allison G, Whittle B, Verma NK: Serotype 1a O-antigen modification: molecular characterization of the genes involved and their novel organization in the Shigella flexneri chromosome. J Bacteriol 1999, 181(15):4711-4718.

6. Allison GE, Verma NK: Serotype-converting bacteriophages and O-antigen modification in Shigella flexneri. Trends Microbiol 2000, 8(1):17-23.

7. Adams MM, Allison GE, Verma NK: Type IV O antigen modification genes in the genome of Shigella flexneri NCTC 8296. Microbiology 2001, 147(Pt 4):851-860.

8. Mavris M, Manning PA, Morona R: Mechanism of bacteriophage Sfllmediated serotype conversion in Shigella flexneri. Mol Microbiol 1997, 26(5):939-950.

9. Allison GE, Angeles D, Tran-Dinh N, Verma NK: Complete genomic sequence of $\mathrm{SfV}$, a serotype-converting temperate bacteriophage of Shigella flexneri. J Bacteriol 2002, 184(7):1974-1987.

10. Casjens S, Winn-Stapley DA, Gilcrease EB, Morona R, Kuhlewein C, Chua JE, Manning PA, Inwood W, Clark AJ: The chromosome of Shigella flexneri bacteriophage Sf6: complete nucleotide sequence, genetic mosaicism, and DNA packaging. J Mol Biol 2004, 339(2):379-394.

11. Allison GE, Angeles DC, Huan P, Verma NK: Morphology of temperate bacteriophage $\mathrm{SfV}$ and characterisation of the DNA packaging and capsid genes: the structural genes evolved from two different phage families. Virology 2003, 308(1):114-127.

12. Guan $\mathrm{S}$, Bastin DA, Verma NK: Functional analysis of the $\mathrm{O}$ antigen glucosylation gene cluster of Shigella flexneri bacteriophage SfX. Microbiology 1999, 145(5):1263-1273.

13. Gemski P Jr, Koeltzow DE, Formal SB: Phage conversion of Shigella flexneri group antigens. Infect Immun 1975, 11(4):685-691.

14. Wei J, Goldberg MB, Burland V, Venkatesan MM, Deng W, Fournier G, Mayhew GF, Plunkett G 3rd, Rose DJ, Darling A, et al: Complete genome sequence and comparative genomics of Shigella flexneri serotype 2a strain 2457T. Infect Immun 2003, 71(5):2775-2786.

15. Jin Q, Yuan Z, Xu J, Wang Y, Shen Y, Lu W, Wang J, Liu H, Yang J, Yang F, et al: Genome sequence of Shigella flexneri 2a: insights into pathogenicity through comparison with genomes of Escherichia coli K12 and O157. Nucleic Acids Res 2002, 30(20):4432-4441

16. Sun Q, Lan R, Wang Y, Wang J, Luo X, Zhang S, Li P, Ye C, Jing H, Xu J: Genesis of a novel Shigella flexneri serotype by sequential infection of serotype-converting bacteriophages SfX and Sfl. BMC Microbiol 2011, 11:269.

17. Bastin DA, Lord A, Verma NK: Cloning and analysis of the glucosyl transferase gene encoding type I antigen in Shigella flexneri. FEMS Microbiol Lett 1997, 156(1):133-139.

18. Stagg RM, Cam PD, Verma NK: Identification of newly recognized serotype $1 \mathrm{c}$ as the most prevalent Shigella flexneri serotype in northern rural Vietnam. Epidemiol Infect 2008, 136(8):1134-1140.

19. Ackermann HW: Tailed bacteriophages: the order caudovirales. Adv Virus Res 1998, 51:135-201.

20. Lindberg AA, Wollin R, Gemski P, Wohlhieter JA: Interaction between bacteriophage Sf6 and Shigella flexner. J Virol 1978, 27(1):38-44.

21. Luo X, Sun Q, Lan R, Wang J, Li Z, Xia S, Zhang J, Wang Y, Jin D, Yuan X, et al: Emergence of a novel Shigella flexneri serotype 1d in China. Diagn Microbiol Infect Dis 2012, 74(3):316-319.

22. Lindberg AA (Ed): Bacterial surface polysaccharides and phage adsorption. New York: Academic; 1977.

23. Campbell AM: Chromosomal insertion sites for phages and plasmids. J Bacteriol 1992, 174(23):7495-7499.

24. Huan PT, Bastin DA, Whittle BL, Lindberg AA, Verma NK: Molecular characterization of the genes involved in O-antigen modification, attachment, integration and excision in Shigella flexneri bacteriophage SfV. Gene 1997, 195(2):217-227.

25. Blattner FR, Plunkett $G$ 3rd, Bloch CA, Perna NT, Burland V, Riley M, Collado-Vides J, Glasner JD, Rode CK, Mayhew GF, et al: The complete genome sequence of Escherichia coli K-12. Science 1997, 277(5331): 1453-1462.

26. Mmolawa PT, Schmieger H, Tucker CP, Heuzenroeder MW: Genomic structure of the Salmonella enterica serovar Typhimurium DT 64 bacteriophage ST64T: evidence for modular genetic architecture. J Bacteriol 2003, 185(11):3473-3475.

27. Guo HC, Kainz M, Roberts JW: Characterization of the late-gene regulatory region of phage 21. J Bacteriol 1991, 173(4):1554-1560. 
28. Schmidt H, Scheef J, Janetzki-Mittmann C, Datz M, Karch H: An ileX tRNA gene is located close to the Shiga toxin II operon in enterohemorrhagic Escherichia coli 0157 and non-O157 strains. FEMS Microbiol Lett 1997, 149(1):39-44.

29. Sambrook J, Fritsch EF, Maniatis T: Molecular Cloning: a Laboratory Manual, 2nd edn. Cold Spring Harbor, NY: Cold Spring Harbor Laboratory; 1989.

doi:10.1186/1471-2180-13-39

Cite this article as: Sun et al:: Isolation and genomic characterization of

$\mathrm{Sfl}$, a serotype-converting bacteriophage of Shigella flexneri. BMC

Microbiology 2013 13:39.

\section{Submit your next manuscript to BioMed Central and take full advantage of:}

- Convenient online submission

- Thorough peer review

- No space constraints or color figure charges

- Immediate publication on acceptance

- Inclusion in PubMed, CAS, Scopus and Google Scholar

- Research which is freely available for redistribution 